\title{
Bloodstream Infections Caused by ESBL-Producing E. coli and K. pneumoniae: Risk Factors for Multidrug-Resistance
}

\author{
Kivanc Serefhanoglu, Hale Turan, Funda Ergin Timurkaynak and Hande Arslan \\ Baskent University, Medical Faculty, Department of Infectious Diseases and Clinical Microbiology; Ankara/Turkey
}

\begin{abstract}
This prospective case-control study was conducted from October 2003 to June 2007 to evaluate risk factors for multidrug resistance among extended-spectrum-b-lactamase-producing Escherichia coli and Klebsiella spp. (ESBLEK) isolates in blood cultures. All adult patients ( $\geq 18$ years old) whose blood cultures grew ESBL-EK during the study period were included. An ESBL-EK isolate was defined as MDR if it was resistant to at least one member of following two classes of antibiotics: aminoglycosides (amikacin, gentamicin, or netilmycin) and fluoroquinolones (ofloxacin, or ciprofloxacin). Case patients were those with a MDR ESBL-EK isolate, and control patients were those with a non-MDR ESBL-EK isolate. A total of 94 bloodstream infections, including 37 (39,4\%) bloodstream infections with ESBL-producing $E$. coli and $57(60,6 \%)$ with ESBL-producing $K$. pneumoniae, in 86 patients were enrolled. Thirty episodes (31.9\%) were due to MDR ESBL-EK. The only independent risk factor for MDR ESBL-EK was duration of hospitalization before bacteraemia (OR 3.88; 95\% CI 1.55-9.71; $\mathrm{p}=0.004$ ). The rate of multidrug resistance among ESBL-EK bloodstream isolates was high, and duration of hospitalization before bacteraemia was the only indeepended risk factor for the MDR ESBL-EK bloodstream infections.
\end{abstract}

Key-Words: ESBLs, multidrug resistance, risk factors.

Extended-spectrum $\beta$-lactamazes (ESBLs) are group of plasmid-borne enzymes with the ability to hydrolyze oxyimino$\beta$-lactams [1]. They are mainly found in a variety of members of the Enterobacteriaceae, predominantly in Escherichia coli (E. coli) and Klebsiella pneumoniae (K. pneumoniae), as well as in Pseudomonas aeruginosa [2,3]. ESBLs confer resistance not only to penicillins, aztreonam, and cephalosporins but could also be resistant to other antibiotic classes including aminoglycosides, trimethoprimsulfamethoxazole, and quinolones [4-6]. The clinical relevance of the multidrug resistance among ESBL-producing E. coli and Klebsiella spp. (ESBL-EK) isolates is of great concern due to the severely limited therapeutic options and increased risk of treatment failure in patients infected with such strains [7]. Therefore it is important to determine risk factors for the infections with multidrug-resistant (MDR) ESBL-EK isolates. Although the phenomenon of antimicrobial coresistance among ESBL-EK isolates of various samples has been reported [8,9], the issue has not been well described among ESBL-EK isolates causing blood stream infections.

In this report, we analyzed the clinical characteristics of the bloodstream infections caused by MDR ESBL-EK isolates, and compared them with those caused by ESBL-EK isolates without such resistance pattern. We also evaluated the antibiotic susceptibility pattern of these ESBL-producing isolates to a variety of antibiotics. In order to avoid the difficulties in differantiation between colonization and infection, only patients' developing bacteraemias were included in the study.

Received on 6 July 2009; revised 22 November 2009.

Address for correspondence: Dr. Kivanc Serefhanoglu. Baskent Universitesi Istanbul Saglik, Uygulama ve Arastirma Hastanesi, Oymaci S. No: 7, 34662 Altunizade-Uskudar/ISTANBUL, TURKEY. Telephone: +90 216 5541500; Fax: 90216 6519858; E-mail: kivanc1972@gmail.com.

The Brazilian Journal of Infectious Diseases 2009;13(6):403-407. (C) 2009 by The Brazilian Journal of Infectious Diseases and Contexto Publishing. All rights reserved.

\section{Material and Methods}

This is a case-control study of bloodstream infections caused by ESBL-EK, and was conducted from October 2003 to June 2007, at Baskent University Konya Medical and Research Center, a 200-bed academic tertiary care center, in Konya, Turkey. The database at the Clinical Microbiology Laboratory and Department of Hospital Infection was prospectively assessed in order to identify patients with ESBLEK bacteremia. The study protocol was approved by the ethics committee of the Baskent University.

An ESBL-EK isolate was defined as MDR if it was resistant to at least one member of following two classes of antibiotics: aminoglycosides (amikacin, gentamicin, or netilmycin) and fluoroquinolones (ofloxacin, or ciprofloxacin). Duplicate isolates from the same patient were excluded. A duplicate isolate was defined as an isolate recovered from blood speciemens less than 15 days apart from the same patient.

In this case-control study, case patients were those with a MDR ESBL-EK isolate, and control patients were those with an ESBL-EK isolate that did not meet criteria for multidrug resistance. All adult patients ( $\geq 18$ years old) whose blood cultures grew ESBL-EK during the study period were included. Nosocomial bloodstream infections as well as other nosocomial infections were defined according to the criteria proposed by the Centers for Disease Control and Prevention [10].

Blood culture samples were collected by peripheral venipuncture and processed in BACTEC 9050, Becton Dickinson, USA. Antimicrobial susceptibility testing of the isolated organisms was done by disk diffusion method using the Kirby-Bauer technique [11], and as per the recommendations of the CLSI (formerly NCCLS) [12]. All disks were obtained from Oxoid limited Wade Road, Basingstoke, Hampshire, RG24 8PW, England. E. coli ATCC 25922 was used as the reference strain for quality control. Drugs tested were ciprofloxacin, ofloxacin, gentamicin, amikacin, netilmicin, piperacillin-tazobactam, cefepime, tetracycline, 
chloramphenicole, trimethoprim-sulfamethoxazole, and imipenem. ESBL production was examined by the combination disk method $[13,14]$. In brief, the diameters of the inhibition zones on cefotaxime and ceftazidime disks ( $30 \mu$ g each), alone and in combination with clavulanic acid $(10 \mu \mathrm{g})$, were determined. An increase in the zone diameter of five $\mathrm{mm}$ or more when either of the antimicrobial agents was combined with clavulanic acid was considered evidence of ESBL production.

Clinical variable collected from patients with bacteraemia included age, gender, the severity of illness, as calculated by the Acute Physiology and Chronic Health Evaluation (APACHE) II score [15], location of the patient in the hospital at the time of bacteremia, underlying medical conditions (including malignancy, chronic renal disease, chronic obstructive pulmonary disease, diabetes mellitus, cerebrovascular disease, and chronic heart failure), laboratory findings, recent hospitalization, duration of hospitalization before onset of bacteremia, insertion of invasive device (i.e., drainage catheter, central venous catheter, mechanical ventilation, and/or urinary catheter), corticosteroid therapy, and any surgical procedure performed during the previous 30 days of onset of bacteremia. Antimicrobial therapy given for at least $48 \mathrm{hr}$ in the last 30 days before onset of bacteraemia was considered as previous antibiotic treatment. Bacteraemia was considered as hospital acquired if the blood culture was collected $>48$ hours after admission of the patient. Death of the patient was attributed to the bacteraemia if there was no other condition documented as cause of death.

\section{Statistical Analysis \\ Collected data were subjected to statistical analysis using SPSS statistical package version 13.0. The risk factors for MDR ESBL-EK were examined by multiple logistic regression analysis and chi-squere test where appropriate. A value of $\mathrm{p}<0.05$ was considered as significant.}

\section{Results}

A total of 94 bloodstream infections, including 37 (39.4\%) bloodstream infections with ESBL-producing E. coli and 57 (60.6\%) with ESBL-producing Klebsiella spp. in 86 patients were enrolled during the study period. Of the 94 episodes, 30 (31.9\%) were due to MDR ESBL-EK. The species of Klebsiella were $K$. pneumoniae in 50 (87.7\%) episodes and $K$. oxytoca in seven (12,3\%). The 30 MDR ESBL-EK isolates included 10 (33.3\%) E. coli, 18 (60\%) K. pneumoniae, and two (6.7\%) K. oxytoca.

The results of multivariate logistic regression analysis for demographics, APACHE II score, nosocomial infection, hospital location at time of infection, duration of hospitalization before bacteremia, surgery in previous 30 days of bacteremia, therapeutic procedures, corticosteroid use in previous 30 days, comorbidities, and antibiotic use within 30 days in case patients with MDR ESBL-EK compared to control group with non-MDR ESBL-EK are shown in Table 1. The only variable, independetly associated with the MDR ESBL-EK infection, was duration of hospitalization before bacteraemia (OR 3.88; 95\% CI 1.55-9.71; $\mathrm{p}=0.004)$. Univariate analysis for the species of infecting organism, mortality, and source of bacteraemia revealed no difference between the two groups (Table 2).

The detail antibiotic resistance pattern of the $E$. coli and Klebsiella spp. isolates is given in Table 3. Only imipenem remained effective against all isolates of ESBL-EK. Strains resistant to piperacillin-tazobactam and cefepime were higher in MDR ESBL-EK group (40 and 70\%, respectively) than in non-MDR ESBL-EK group (35.9 and 64.1\%, respectively); about $80 \%$ of isolates in the both groups were resistant to trimethoprim-sulfamethoxazole; 39.1 and $60.9 \%$ of non-MDR ESBL-EK strains displayed resistance against at least one member of aminoglycosides and fluoroquinolones tested, respectively; and tetracycline remained largely (approximately 94\%) resistant among strains in the both groups.

\section{Discussion}

The presenting study investigated bloodstream infections due to ESBL-EK in our center over a three and one-half years period. A total of 94 strains were obtained in which a high rate (31.9\%) of multidrug resistance (ESBL-EK with both an aminoglycoside and a quinolone resistance) was detected. Since plasmids frequently carry both the ESBL and aminoglycoside resistance genes and many Enterobacteriacea species have also chromosomal resistance to quinolones, the ESBL-producing Enterobacteriacea are commonly multidrug resistant [16]. Our finding is in accordonce with several previous researchs that also found a high proportion of multidrug resistance among ESBL producers. Paterson et al. reported a close relationship between ESBL production and ciprofloxacin resistance in K. pneumoniae [6]. A high rate (18 to $59.5 \%$ ) of a quinolone [6,17-21] and/or an aminoglycoside (63-77\%) [18,22] resistance has been reported among ESBL producers from the various geopraphical areas.

We used a case-control approach to identify clinical predictors of bacteraemia involving MDR ESBL-EK organisms. It has been suggested that risk factors for an infection may differ from risk factors for colonization [23]. So, our study is important since we studied true infections by including only bloodstream isolates. We found that duration of hospitalization before bacteraemia (OR 3.88; 95\% CI 1.559.71; $\mathrm{p}=0.004$ ) was the only predictor of bacteraemia due to a MDR ESBL-EK. This indentified risk factor is not surprising. In the current study, the mean hospital stay before bacteremia was 16.6 days in patients who developed MDR ESBL-EK bacteraemia versus eight days in those who had non-MDR ESBL-EK bacteremia. It is well known that K. pneumoniae primarily attacks such individuals who are hospitalized for severe underlying diseases. Those patients often require prolonged hospitalization and invasive procedures, which breach the normal host defenses and increase the risk of bacterial colonization and infection [24]. It is not an unexpected scenario that the patients with long hospitalization require 
Table 1. Risk factors for MDR ESBL-EK in bloodstream infections, n (\%)*.

\begin{tabular}{|c|c|c|c|c|}
\hline & $\begin{array}{l}\text { MDRESBL-EK } \\
\operatorname{group}(n=30)\end{array}$ & $\begin{array}{l}\text { Non-MDRESBL-EK } \\
\text { group }(n=64)\end{array}$ & OR (95\% CI) & pvalue \\
\hline \multicolumn{5}{|l|}{ Demographics } \\
\hline Age in years, mean SD & $65.8 \pm 16.3$ & $62 \pm 16.4$ & $1.01(0.96-1.07)$ & 0.658 \\
\hline Sex & & & $2.07(0.28-15.38)$ & 0.479 \\
\hline Male & $18(60)$ & 35 54.7) & & \\
\hline Female & $12(40)$ & 29 (45.3) & & \\
\hline APACHE II, mean \pm SD & $16.2 \pm 9.8$ & $15.6 \pm 9.3$ & $0.91(0.78-1.07)$ & 0.259 \\
\hline Nosocomial infection & $29(96.7)$ & $61(95.3)$ & $8.52(0.00-2.70)$ & 0.779 \\
\hline Hospital location at time of infection & & & $0.00(0.00-)$ & 0.999 \\
\hline Intensive care unit & $25(86.2)$ & $44(72.1)$ & & \\
\hline General medical or surgical ward & $4(13.8)$ & 17 (27.9) & & \\
\hline $\begin{array}{l}\text { Hospital stay before bacteremia, } \\
\text { mean days (range) }\end{array}$ & $16.6(7-79)$ & $8(2-16)$ & $3.88(1.55-9.71)$ & 0.004 \\
\hline Surgical procedure in previous 30 days & $8(26.7)$ & $16(25)$ & $0.76(0.06-10.06)$ & 0.834 \\
\hline Mechanical ventilation & $12(40)$ & $16(25)$ & $0.19(0.02-1.95)$ & 0.163 \\
\hline Urinary catheterization & 28 (93.3) & $60(93.8)$ & $0.22(0.00-18.69)$ & 0.507 \\
\hline Central venous catheter & 22 (73.3) & 46 (71.9) & $1.04(0.08-13.46)$ & 0.977 \\
\hline Drainage catheter & 5 (16.7) & $8(12.5)$ & $2.74(0.16-46.01)$ & 0.483 \\
\hline Nursing home resident & - & $2(3.1)$ & 4.3E $(0.00-)$ & 0.999 \\
\hline Corticosteroid use in previous $30 \mathrm{~d}$ & $3(10)$ & $8(12.5)$ & $1.91(0.03-143.53)$ & 0.768 \\
\hline \multicolumn{5}{|l|}{ Underlying diseases } \\
\hline Diabetes mellitus & 8 (26.7) & $16(25)$ & $1.96(0.14-26.62)$ & 0.613 \\
\hline Chronic obstructive pulmonary disease & 5 (16.7) & $14(21.9)$ & $2.10(0.16-27.57)$ & 0.571 \\
\hline Cerebrovascular disease & 7 (23.3) & $13(20.3)$ & $1.82(0.12-24.76)$ & 0.598 \\
\hline Chronic renal disease & 5 (16.7) & $8(12.5)$ & $1.46(0.05-41.68)$ & 0.824 \\
\hline Malignancy & $1(3.3)$ & $4(6.3)$ & $2.35(0.02-269.85)$ & 0.725 \\
\hline Chronic heart failure & $2(6.7)$ & $5(7.8)$ & $0.38(0.01-13.85)$ & 0.597 \\
\hline $\begin{array}{l}\text { Prior use of any antibiotic } \\
\text { within previous } 30 \mathrm{~d}\end{array}$ & $29(96.7)$ & $58(90.6)$ & $3.00(0.34-26.10)$ & 0.298 \\
\hline \multicolumn{5}{|c|}{ Prior use of spesific antibiotic within previous $30 \mathrm{~d}$} \\
\hline Trimethoprim-sulfamethoxazole & $4(13.3)$ & $5(7.8)$ & $0.89(0.04-20.91)$ & 0.943 \\
\hline Broad-spectrum cephalosporin ${ }^{\mathrm{a}}$ & 11 (36.7) & $25(39.1)$ & $1.64(0.12-21.65)$ & 0.707 \\
\hline Narrow-spectrum cephalosporin ${ }^{\mathrm{b}}$ & $5(16.7)$ & $8(12.5)$ & $11.10(0.63-194.68)$ & 0.100 \\
\hline Extended-spectrum penicillins ${ }^{\mathrm{c}}$ & $6(20)$ & $17(26.6)$ & $1.23(0.14-10.59)$ & 0.853 \\
\hline Piperacillin-tazobactam & 4 (13.3) & $7(10.9)$ & $2.30(0.19-27.69)$ & 0.513 \\
\hline Carbapenems $^{\mathrm{d}}$ & $4(13.3)$ & $6(9.4)$ & $0.56(0.01-30.67)$ & 0.777 \\
\hline Aminoglycosides ${ }^{\mathrm{e}}$ & $3(10)$ & $6(9.4)$ & $0.02(0.00-9.03)$ & 0.209 \\
\hline Quinolones $^{\mathrm{f}}$ & $13(43.3)$ & $22(34.4)$ & $0.17(0.02-1.36)$ & 0.094 \\
\hline
\end{tabular}

* Logistic regression analysis is used for analysis; a Includes ceftazidime, cefotaxime, ceftriaxone, and cefepime; ${ }^{\mathrm{b}}$ Includes cefozolin, cefuroxime; ' Includes ampicillinsulbactam, amoxicillin-clavulanate; ${ }^{\mathrm{d}}$ Includes meropenem, imipenem; ${ }^{\mathrm{e}}$ Includes amikacin, gentamicin, and netilmycin; ${ }^{\mathrm{f}}$ Includes ciprofloxacin, ofloxacin.

Table 2. Source, infecting organism, and mortality in the MDR ESBL-EK and non-MDR ESBL-EK groups, $\mathrm{n}(\%)^{*}$.

\begin{tabular}{|c|c|c|c|}
\hline & MDRESBL-EK group $(n=30)$ & Non-MDR ESBL-EK group $(n=64)$ & pvalue \\
\hline Sources of bacteremia & & & 0.777 \\
\hline Urinary & $11(36.7)$ & $26(40.6)$ & \\
\hline Pneumonia & 7 (23.3) & $11(17.2)$ & \\
\hline Skin/soft tissue & $3(10)$ & $5(7.8)$ & \\
\hline Abdominal & - & $3(4.7)$ & \\
\hline Venous catheter & $3(10)$ & $4(6.2)$ & \\
\hline Unknown & $6(20)$ & $15(23.5)$ & \\
\hline Organism & & & 0.413 \\
\hline E. coli & $10(33.3)$ & $27(42.2)$ & \\
\hline Klebsiella spp. & $20(66.7)$ & 37 (57.8) & \\
\hline Mortality & 7 (23.3) & 12 (18.8) & 0.606 \\
\hline
\end{tabular}

${ }^{*}$ Chi-squere test is used for analysis. 
Table 3. Resistance pattern of bloodstream MDR-and-non-MDR ESBL-EK isolates to various antimicrobial agents.

\begin{tabular}{lcrc}
\hline & \multicolumn{3}{c}{ No. (\%) of isolates with resistance to drug } \\
\cline { 2 - 4 } Antibiotic & MDR ESBL-EK strains (n=30) & Non-MDR ESBL-EK strains (n=64) & All (n=94) \\
\hline Aminoglycosides $^{\mathrm{a}}$ & $30(100)$ & $25(39.1)$ & $55(58.5)$ \\
Fluoroquinolones $^{\mathrm{b}}$ & $30(100)$ & $39(60.9)$ & $69(73.4)$ \\
Trimethoprim-sulfamethoxazole $_{\text {Imipenem }}$ & $25(83.3)$ & $52(81.3)$ & $77(81.9)$ \\
Piperacillin-tazobactam & - & - & - \\
Cefepime & $12(40)$ & $23(35.9)$ & $35(37.2)$ \\
Tetracycline & $21(70)$ & $41(64.1)$ & $62(65.9)$ \\
Chloramphenicole & $28(93.3)$ & $61(95.3)$ & $89(94.7)$ \\
\hline
\end{tabular}

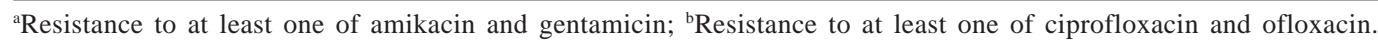

frequent antibiotic therapy, and in turn antibiotic therapy promotes colonization or infection of the treated patients with resistant organisms by eradicating susceptible isolates and reducing the host's resistance to colonization [18]. Furthermore, review of literature shows that hospitalization for a long period of time is a risk factor for the isolation of various highly resistant organisms [18,25-27]. In our study, while prolonged hospitalization was a risk factor for bacteraemia due to a MDR ESBL-EK, APECHE II score, used to assess severity of the patients, was not. It would be proposed that patients who need prolonged hospitalization are expected to have higher APECHE II scores. A possible explanation for this discrepancy may be that the patients in this study were generally medically debilitated patients, who may frequently need hospitalization for a long time due to their underlying diseases but can have a relatively low APECHE II score. In addition, a severe disease does not necessitate the existence of a high APECHE II score. APECHE II score shows severity of the status of the patient at the time of admission to an intensive care unit but not the presence of a severe disease or general illness status.

Antibiotic resistance to the various antibiotics tested was striking in the present study and comparable between MDR ESBL-EK and non-ESBL-EK isolates (Table 3). Among all of 94 isolates, $89.4 \%$ were resistant to tetracycline, $75.5 \%$ to a fluroquinolone tested, $86.2 \%$ to trimethoprimsulfamethoxazole, $69.1 \%$ to piperacillin-tazobactam and $67 \%$ to cefepime. Our data means that although the use of cefepime or piperacillin-tazobactam to treat ESBL-EK infections (when an ESBL-EK is susceptible in vitro) are controversial [28,29], the use of these antibiotics for an early, empiric therapy may result in failure in a significant proportion of patients. In general, the resistance rates to these antibiotics among isolates in our study are similar to that reported from Israel [30] and Colombia [31], but higher than that in USA [7,32] and Singapore [33].

In conclusion, the rate of multidrug resistance among ESBL-EK bloodstream isolates was high at the Baskent University Konya Medical and Research Center, which is a serious threat to antibiotic therapy and may increase the rate of treatment failure. Duration of hospitalization before bacteraemia was the only independed risk factor for the MDR ESBL-EK bloodstream infections.

\section{References}

1. Bush K. Is it important to identify extended spectrum $\beta$-lactamase producing isolates? Eur J Clin Microb Infect Dis. 1996;15:361364.

2. Jeong SH, Bae IK, Kwon SB, et al. Investigation of extendedspectrum $\beta$-lactamases produced by clinical isolates of Klebsiella pneumoniae and Escherichia coli in Korea. Lett Appl Microbiol. 2004;39:41-47.

3. Mendelson G, Hait V, Ben-Israel J, et al. Prevalence and risk factors of extended-spectrum beta-lactamase-producing Escherichia coli and Klebsiella pneumoniae in an Israeli long-term care facility. Eur J Clin Microbiol Infect Dis. 2005;24:17-22.

4. Paterson DL, Bonomo RA. Extended-spectrum beta-lactamases: a clinical update. Clin Microbiol Rev. 2005;18:657-686.

5. Oteo J, Campos J, Baquero F. Antibiotic resistance in 1962 invasive isolates of Escherichia coli in 27 Spanish hospitals participating in the European Antimicrobial Resistance Surveillance System. J Antimicrob Chemother. 2001;50:945-952.

6. Paterson DL, Mulazimoglu L, Casellas JM, et al. Epidemiology of ciprofloxacin resistance and its relationship to extendedspectrum beta-lactamase production in Klebsiella pneumoniae isolates causing bacteremia. Clin Infect Dis. 2000;30:473-478.

7. Hyle EP, Lipworth AD, Zaoutis TE, et al. Risk Factors for Increasing Multidrug Resistance among Extended-Spectrum $\beta$ Lactamase-Producing Escherichia coli and Klebsiella Species. Clin Infect Dis. 2005;40:1317-1324.

8. Bell JM, Turnidge JD, Gales AC, et al. Prevalence of extended spectrum beta-lactamase (ESBL)-producing clinical isolates in the Asia-Pacific region and South Africa: regional results from SENTRY Antimicrobial Surveillance Program (1998-99). Diagn Microbiol Infect Dis. 2002;42:193-198.

9. Spanu T, Luzzaro F, Perilli M, et al. and The Italian ESBL Study Group. Occurrence of extended-spectrum $\beta$-lactamases in members of the family Enterobacteriaceae in Italy: implications for resistance to $\beta$-lactams and other antimicrobial drugs. Antimicrob Agents Chemother. 2002;46:196-202.

10. Garner JS, Jarvis WR, Emori TG, et al. CDC definitions for nosocomial infections. Am J Infect Control. 1988;16:128-140.

11. Baur AW, Kirby WMM, Sherris JC, et al. Antibiotic susceptibility testing by a standardized single disk method. Am J Clin Path. 1966;45:493-496.

12. Performance standards for antimicrobial susceptibility testing; ninth informational supplement, 1999;M100-S9. National Committee for Clinical Laboratory Standards, Wayne, PA7.

13. Jacoby, GA, Han P. Detection of extended-spectrum $\beta$-lactamases in clinical isolates of Klebsiella pneumoniae and Escherichia coli. J Clin Microbiol. 1996;34:908-911. 
14. Performance standards for antimicrobial susceptibility testing; fourteenth informational supplement, 2004;M100-S14, p. 24, Table 2A. National Committee for Clinical Laboratory Standards, Wayne, Pa.

15. Knaus WA, Drapier EA, Wagner DP, et al. APACHE II: a severity of disease classification system. Crit Care Med. 1985;13:818829.

16. Paterson DL. Resistance in Gram-negative bacteria: Enterobacteriaceae. Am J Infect Control. 2006;34:20-28.

17. Villegas MV, Correa A, Perez F, et al. the Colombian Nosocomial Resistance Study Group. Prevalence and characterization of extended-spectrum $\beta$-lactamases in Klebsiella pneumoniae and Escherichia coli isolates from Colombian Hospitals. Diagn Microbiol Infect Dis. 2004;49:217-222.

18. Tumbarello M, Spanu T, Sanguinetti M, et al. Bloodstream infections caused by extended-spectrum- $\beta$-lactamase-producing Klebsiella pneumoniae: Risk Factors, molecular epidemiology, and clinical outcome. Antimicrob Agents and Chemother. 2006;50:498-504.

19. Itokazu GS, Quinn JP, Bell-Dixon C, et al. Antimicrobial resistance rates among aerobic Gram-negative bacilli recovered from patients in intensive care units: evaluation of a national postmarketing surveillance program. Clin Infect Dis. 1996;23:779-784.

20. Lautenbach EBL, Bilker SWB, Patel JB, et al. Epidemiological investigation of fluoroquinolone resistance in infections due to extended-spectrum $\beta$-lactamase-producing Escherichia coli and Klebsiella pneumoniae. Clin Infect Dis. 2001;33:1288-1294.

21. Yu WL, Jones RN, Hollis RJ, et al. Molecular epidemiology of extended-spectrum $\beta$-lactamase producing, fluoroquinoloneresistant isolates of Klebsiella pneumoniae in Taiwan. J Clin Microbiol. 2002;40:4666-4669.

22. Ben-Ami R, Schwaber MJ, Navon-Venezia S, et al. Influx of extended-spectrum $\beta$-lactamase-producing enterobacteriaceae into the hospital. Clin Infect Dis. 2006;42:925-934.

23. Lucet JC, Chevret S, Decre D, et al. Outbreak of multiply resistant Enterobacteriaceae in an intensive care unit: epidemiology and risk factors for acquisition. Clin Infect Dis. 1996;22:430-436.
24. Podschun, R, Ullmann U. Klebsiella spp. as nosocomial pathogens: epidemiology, taxonomy, typing methods, and pathogenicity factors. Clin Microbiol Rev. 1998;11:589-603.

25. Falagas ME, Bliziotis IA. Pandrug-resistant Gram-negative bacteria: the dawn of the post-antibiotic era? Int J Antimicrob Agents. 2007;29:630-636.

26. Shah AA, Hasan F, Ahmed S, et al. Characteristics, epidemiology and clinical importance of emerging strains of Gram-negative bacilli producing extended-spectrum $\beta$-lactamases. Res Microbiol. 2004;155:409-421.

27. Marra AR, Wey SB, Castelo A, et al. Nosocomial bloodstream infections caused by Klebsiella pneumoniae: impact of extendedspectrum $\beta$-lactamase (ESBL) production on clinical outcome in a hospital with high ESBL prevalence. BMC Infect Dis. 2006;6:24-32.

28. Paterson DL. Recommendation for treatment of severe infections caused by Enterobacteriaceae producing extended-spectrum $\beta$ lactamases (ESBLs). Clin Microbiol Infect. 2000;6:460-463.

29. Yu WL, Pfaller MA, Winokur PL, et al. Cefepime MIC as a predictor of the extended-spectrum $\beta$-lactamase type in Klebsiella pneumonia. Emerg Infect Dis. 2002;8:522-524.

30. Schwaber MJ, Navon-Venezia S, Schwartz D, et al. High Levels of Antimicrobial Coresistance among Extended-Spectrum- $\beta$ Lactamase-Producing Enterobacteriaceae. Antýmicrob Agents and Chemother. 2005;49:2137-2139.

31. Villegasa MV, Correaa A, Pereza F, et al. Prevalence and characterization of extended-spectrum $\beta$-lactamases in Klebsiella pneumoniae and Escherichia coli isolates from Colombian hospitals. Diagn Microbiol Infect Dis. 2004;49:217-222.

32. DiPersioa JR, Deshpandeb LM, Biedenbachb DJ, et al. Evolution and dissemination of extended-spectrum $\beta$-lactamase-producing Klebsiella pneumoniae: Epidemiology and molecular report from the SENTRY Antimicrobial Surveillance Program (1997-2003). Diagn Microbiol Infect Dis. 2005;51:1-7.

33. Chlebicki MP, Oh HML. Extended-spectrum $\beta$-lactamases in clinical isolates of E. coli and Klebsiella spp. in a Singopore hospital: Clinical spectrum. Ann Acad Med Singopore. 2004;33:302-306. 